\title{
Dislocations emission and crack extension at the atomistic crack tip in body-centered-cubic metal Mo
}

\author{
Qiheng Tang and Tzuchiang Wang \\ LNM Institute of Mechanics, Chinese Academy of Sciences, \\ Beijing 100080, People's Republic of China
}

(Received 11 June 1998; accepted 7 July 1999)

\begin{abstract}
The behaviors of a crack in body-centered-cubic metal Mo under different loading modes were studied using the molecular dynamics method. Dislocation emission was observed near the crack tip in response to mode II loading with $\theta=0^{\circ}$ in which $\theta$ is the inclination angle of the slip plane with respect to the crack plane, and two full dislocations were observed at the stress level of $K_{\mathrm{II}}=1.17 \mathrm{MPa} \mathrm{m}^{1 / 2}$ without any evidence of crack extension. Within the range of $0^{\circ} \leqslant \theta \leqslant 45^{\circ}$, crack extension was observed in response to mode I loading, and the effect of crystal orientation on the crack propagation was studied. The crack propagated along the [111] slip direction without any evidence of dislocations emission.
\end{abstract}

\section{INTRODUCTION}

This paper is concerned with the topic of the brittle versus ductile behavior of materials which is still one of both fundamental and technological relevance. While the brittle fracture is well understood in terms of the Griffith criterion, ${ }^{1}$ ductile fracture involves dislocation nucleation and its subsequent motion away from crack tip., ${ }^{2,3}$ The response of brittle versus ductile for a crack depends upon many factors such as temperature, loading rate, loading modes, and crystal orientation. ${ }^{4-8}$ The experimental results obtained by Wang and Anderson ${ }^{5}$ indicated that for a copper bicrystal containing Bi impurity the ductile or brittle response of an interfacial crack is strongly dependent on the relative orientation of available slip planes to the existing crack plane and growth direction but less dependent on the level of the impurity at interface. A four-point bending experiment was conducted by Hiroaki et al. ${ }^{6}$ at $77 \mathrm{~K}$ for Mo bicrystals with various misorientations from $0^{\circ}$ to $90^{\circ}$, and a stressstrain curve to fracture was measured as a function of misorientation angle. The grain boundaries in the range of $13-40^{\circ}$ were very weak and the fracture stress was about $1000 \mathrm{MPa}$, whereas those around $70^{\circ}$ and $89^{\circ}$ were strong and showed a peak of $1700 \mathrm{MPa}$ for fracture stress. Beltz and $\mathrm{Wang}^{7}$ pointed out that the competition between dislocation emission and cleavage decohesion controls the ductile versus brittle behavior of a metal/ ceramic interface and the crack response is directiondependent. In situ transmission electron microscopy (TEM) observation experiments of crack behaviors in body-centered-cubic (bcc) Mo were is carried out by Zhang et al., ${ }^{8}$ and alternating brittle crack propagation and dislocation emission were observed. Their analysis revealed that dislocation emission and crack propagation are closely associated with a mixed-mode intergranular crack and the orientation of tensile axis. Theoretical analysis progress of dislocation nucleation from a crack tip has been made by Schoeck, ${ }^{9}$ Rice, ${ }^{10}$ and others. ${ }^{11,12}$ On the basis of hybrid continuum-atomistic approach elasticity, Rice ${ }^{10}$ and Rice and Beltz ${ }^{11}$ reanalyzed the Rice-Thomson criterion. ${ }^{13}$ For the mode II case, they obtained an exact solution for dislocation nucleation within Peierls framework and proposed a new solid-state parameter, the unstable stacking energy $\gamma_{\mathrm{us}}$ which characterizes the resistance to dislocation nucleation. Wang ${ }^{12}$ extended Rice's analysis with a set of new governing equations which can be employed to analyze the dislocation nucleation and emission. The mixed-mode loads were considered in his analysis. Explicit formulae were proposed for the critical condition of crack cleavage propagation after a number of dislocations emission. But these analyses above did not consider the effect of ledge formation. Using a variational boundary integral method which was developed in Ref. 14 , Xu et al. ${ }^{15,16}$ calculated several activation configurations of embryonic dislocation nucleated from a crack tip. The configurations considered by $\mathrm{Xu}$ et al. ${ }^{15,16}$ included nucleation of dislocation on extension of the crack surface, nucleation on an inclined plane containing the crack front, nucleation on an oblique plane, and nucleation on a cleavage ledge. Their calculation results clearly show that nucleation of dislocations from crack tip in intrinsically brittle crystals is the most favorable at local crack front heterogeneities such as cleavage ledges. Similar result has been obtained by Zhou et al., ${ }^{17}$ Orloy, ${ }^{18}$ and others. ${ }^{19,20}$ On the basis of the molecular dynamics method, Cleri et al. ${ }^{19}$ simulated dislocation emission on an inclined plane and obtained the atomic level displacement and stress fields. Their atomistic simulation suggested that 
nucleation of dislocation on an inclined plane from crack tip is intimately connected with the formation of surface steps.

Due to the nonlinearity and atomic lattice effects, the continuum elasticity is not suitable for describing the deformation behaviors very near the crack tip. So it is essential to analyze the dislocation nucleation and crack propagation in the crack tip region by atomic simulation. The different loading modes and crystal orientation were considered by the following researchers. Mullins ${ }^{21}$ examined the behavior of a crack of iron crystal at the atomic scale under mixed-mode loads. The crack tip deformation in $\mathrm{Al}$ was investigated by Hoagland et al. $^{22}$ using an embedded atom potential; three crack/crystal orientations were examined, and two of them were in a pure brittle mode without dislocation emission. The ductile versus brittle behaviors at an atomic crack tip in metal copper were simulated by Zhang et al. ${ }^{23}$ Some interesting results were obtained in their simulation, such as crack cleavage propagation, cleavage following dislocation emission, and blunting or superblunting by the emitted dislocations.

In the present paper, we use atomistic molecular dynamics simulation to test the validity of models based on continuum elasticity that predicts brittle versus ductile response of a crack in pure metal Mo. As we shall discuss below, our simulation results suggest strongly that the brittle versus ductile response of metal Mo depends on the loading modes and the relative orientation of the available slip plane to the crack plane and growth direction.

\section{CALCULATION METHOD}

\section{A. Interatomic potential}

The " $N$-body" potential proposed by Tang et al. ${ }^{24}$ is used to describe the interatomic interaction. The basic equation of the embedded atom method $(\mathrm{EAM})^{25}$ is

$$
E_{T}=1 / 2 \sum_{i, j, i \neq j} \phi\left(r_{i j}\right)+\sum_{i} F\left(\frac{\rho_{i}}{\rho_{e}}\right)
$$

where $E_{T}$ is the total energy of an assembly of atoms, $r_{i j}$ is the separation distance between atoms $i$ and $j, \rho_{\mathrm{i}}$ and $\rho_{e}$ are the electron densities at any atom $i$ in the deformed and undeformed states, respectively, $\phi\left(r_{i j}\right)$ is the pair potential, and $F\left(\rho_{i} / \rho_{e}\right)$ is the embedding energy function.

$$
\rho_{i}\left(r_{i j}\right)=\sum_{j, j \neq i} \rho_{j}^{a}\left(\frac{r_{1 e}}{r_{i j}}\right)
$$

where $r_{1 e}$ and $r_{2 e}$ are the first- and second-neighbor atom distance, respectively.
For a monoatom metal, a simple formula for $\rho_{j}^{a}$ proposed by Johnson ${ }^{26}$ is adopted in our analysis,

$$
\rho_{j}^{a}\left(r_{i j}\right)=\left(\frac{r_{1 e}}{r_{i j}}\right)^{\beta},
$$

where $\beta=5.029 .^{24}$

On the basis of Johnson's work, ${ }^{26}$ an " $N$-body" potential constructed by Tang et al. ${ }^{24}$ is used to describe the interatomic interactions in this paper. Some constants of bcc metal Mo were used to construct the " $N$-body" potential. See Table I, where $E_{I V}^{U F}$ is vacancy formation energy, $a_{0}$ is the lattice constant, $E_{c}$ is the cohesive energy, and $\mu$ is the Voigt average shear modulus; $\mu=(3 C+$ $\left.2 C^{\prime}\right) / 5, C=C_{44}$, and $C^{\prime}=\left(C_{11}-C_{12}\right) / 2 . \phi\left(r_{i j}\right)$ is a two-body central potential between atoms $i$ and $j$, and $F\left(\rho_{i} / \rho_{e}\right)$ is the embedding function which was obtained by means of a numerical experiment for uniaxial tension. They are described by the following formulas:

$$
\begin{aligned}
\phi\left(\frac{r_{i j}}{r_{1 e}}\right)= & B_{0}+B_{1}\left(\frac{r_{i j}}{r_{1 e}}-1\right)+B_{2}\left(\frac{r_{i j}}{r_{1 e}}-1\right)^{2} \\
& +B_{3}\left(\frac{r_{i j}}{r_{1 e}}-1\right)^{3}+H\left(r_{i j}-r_{2 e}\right)\left[B_{4}\left(\frac{r_{i j}}{r_{1 e}}-1\right)^{3}\right. \\
& \left.+B_{5}\left(\frac{r_{i j}}{r_{1 e}}-1\right)^{3}\left(\frac{r_{c}}{r_{2 e}}-1\right)\right] /\left(\frac{r_{c}}{r_{2 e}}-1\right)^{3},
\end{aligned}
$$

$F^{\prime}\left(\frac{\rho}{\rho_{e}}\right)=$

$$
\left\{\begin{array}{c}
-10 \sqrt{0.0515-\left(\frac{\rho}{\rho_{e}}-0.778\right)^{2}}+1.9 \\
0.68 \leqslant \frac{\rho}{\rho_{e}} \leqslant 0.775 \\
-0.72\left(\frac{\rho}{\rho_{e}}-0.775\right)-0.3 \\
0.775 \leqslant \frac{\rho}{\rho_{e}} \leqslant 0.83 \\
-10 \sqrt{0.397-\left(\frac{\rho}{\rho_{e}}-0.88\right)^{2}}+0.59 \\
0.83 \leqslant \frac{\rho}{\rho_{e}} \leqslant 1.01
\end{array}\right.
$$

TABLE I. Values of the lattice constant, the cohesive energy, and vacancy formation energy are the same as that of Johnson. ${ }^{26}$ The elastic constants are from Ref. 27. The coefficients $B_{\mathrm{i}}(i=0, \ldots 5)$ are for the pair potential, $n$, and $r_{c}$ is the cutoff distance for the potential which is between the second- and third-neighbor distances.

\begin{tabular}{ccccccc}
\hline \hline $\begin{array}{c}a_{0} \\
(\AA)\end{array}$ & $\begin{array}{c}E_{c} \\
(\mathrm{eV})\end{array}$ & $\begin{array}{c}E_{I V}^{U F} \\
(\mathrm{eV})\end{array}$ & $\begin{array}{c}\mu \\
(\mathrm{GPa})\end{array}$ & $\begin{array}{c}C_{11} \\
(\mathrm{GPa})\end{array}$ & $\begin{array}{c}C_{12} \\
(\mathrm{GPa})\end{array}$ & $\begin{array}{c}C_{44} \\
(\mathrm{GPa})\end{array}$ \\
\hline 3.150 & 6.810 & 3.40 & 124.8 & 459 & 168 & 111 \\
\hline \hline $\begin{array}{c}B_{0} \\
(\mathrm{eV})\end{array}$ & $\begin{array}{c}B_{1} \\
(\mathrm{eV})\end{array}$ & $\begin{array}{c}B_{2} \\
(\mathrm{eV})\end{array}$ & $\begin{array}{c}B_{3} \\
(\mathrm{eV})\end{array}$ & $\begin{array}{c}B_{4} \\
(\mathrm{eV})\end{array}$ & $\begin{array}{c}B_{5} \\
(\mathrm{eV})\end{array}$ & $\begin{array}{c}r_{c} \\
\left(a_{0}\right)\end{array}$ \\
\hline-1.650 & 11.580 & -0.325 & -0.450 & -1.060 & 3.800 & 1.250 \\
\hline \hline
\end{tabular}


The coefficients $B_{i}(i=0, \ldots 5)$ in Eq. (4) were given in Table I; see Ref. 24. $H\left(\tau_{i j}-\tau_{2 e}\right)$ is the step function.

\section{B. Crack tip geometry}

The parallelepiped with a slit is used as the simulation model in which the slip plane is coincident with crack plane $\left(\theta=0^{0}\right)$. The coordinate system is selected to be the $x, y$, and $z$ axes along [111], [110], and [112] , respectively. As shown in Figs. 1(a)-1(c), the size of the atomic area is $500 \times(\sqrt{3} / 6) a_{0}$ in the $x$ direction, $40 \times(\sqrt{2} / 2) a_{0}$ in the $y$ direction, and $6 \times(\sqrt{6} / 6) a_{0}$ in the $z$ direction. Along the $x$ direction, the periodicity is in three layers of (111) atomic planes and its length is $3 \times(\sqrt{3} / 6) a_{0}$, along the $y$ direction, the periodicity is in two layers of the (110) atomic plane and its length is $2 \times(\sqrt{2} / 2) a_{0}$, and along the $z$ direction, the periodicity is six layers of the $(11 \overline{2})$ atomic plane and its length is $6 \times(\sqrt{6} / 6) a_{0}$. The left side of boundary to the crack tip is $120 \times(\sqrt{3} / 6) a_{0}$, the separation of the upper and lower crack planes is taken to be $\sqrt{2} a_{0}$, and the number of atoms in the present model is about 20,000. Figure 1(c) shows the atomic arrangement

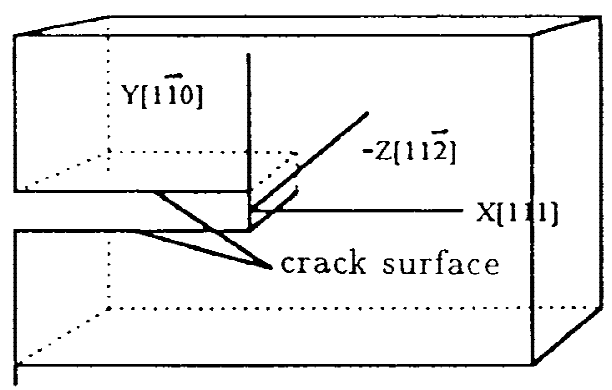

(a)

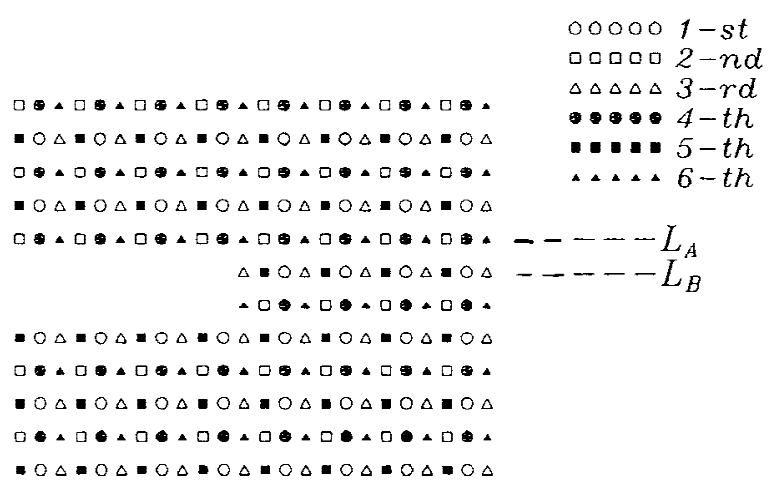

(c)
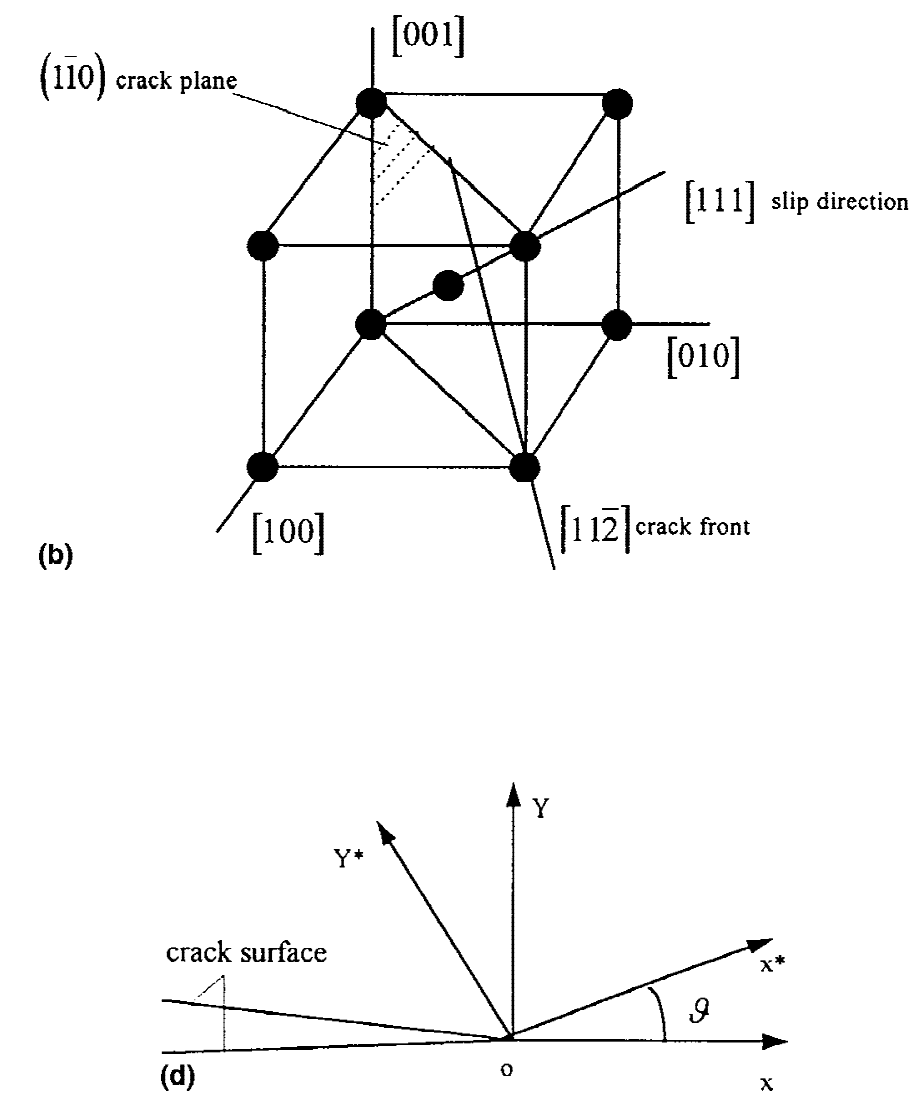

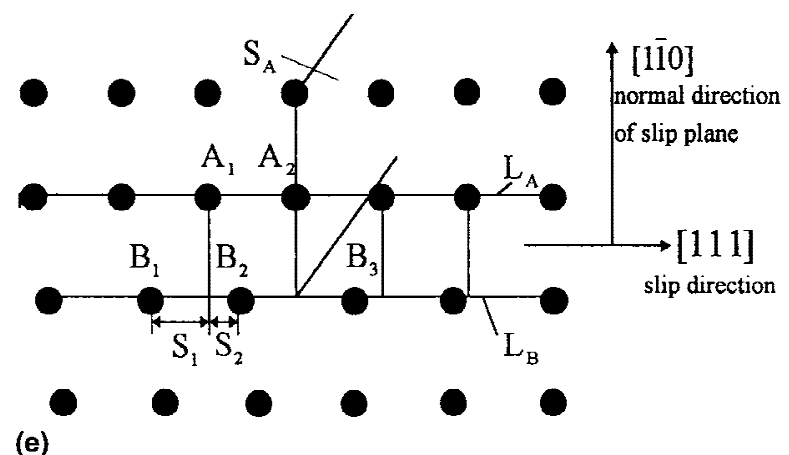

(e)

FIG. 1. Crack tip geometry in bcc metal Mo: (a) schematic diagram for simulation model; (b) bcc lattice in relation to the crack plane, crack front, and slip direction; (c) initial positions of atomic arrangement near the crack tip region, with six layers of (11 $\overline{2})$ planes projected on the same plane; (d) relation of the coordinate systems of $X O Y$ and $X^{*} O Y^{*}$; (e) schematic diagram of atomic area in the deformed state. 
of six adjacent $(11 \overline{2})$ planes projected on the same layer; $L_{A}$ and $L_{B}$ in Figs. 1(c) and 1(e) are the two adjacent (110) atomic planes.

As $\theta$ varies, the $z$ axis is still along [112] , but directions of axes $x$ and $y$ are changed; see Fig. 1(d). The change of the number of atoms is quite small, and the total number of atoms is still about 20,000.

The effects of crack tip geometry on dislocation emission and cleavage have been studied by Schiøtz et al. ${ }^{20}$ and Gumbsch. ${ }^{28}$ The results given by Gumbsch ${ }^{28}$ show that critical intensity factors required to propagate the crack or to emit dislocation from the crack tip modestly increased as the crack is blunted. A similar tendency were observed by Schiøtz et al. ${ }^{20}$ However, Schiøtz et $a .^{20}$ pointed out that for some materials blunting changes the preferred deformation mode from brittle cleavage to dislocation emission. In such materials, the absorption of preexisting dislocations by the crack tip can cause the crack tip to be locally arrested, causing a significant increase in the microscopic toughness of the crack tip. In our simulation we use a slight blunted crack tip instead of a sharp crack. On the basis of a study by Gumbsch, ${ }^{28}$ we can presume that such a choice of crack tip geometry only has a marginal effect on the simulation results. The changes of deformation mode from brittle cleavage to ductile for metal Mo in the present simulation seems not possible. The reason will be explained in Sec. III.

\section{Boundary condition and loading method}

The fixed displacement boundary scheme is applied with the mode II (or I) singularity field of the plane strain for an isotropic elastic continuum. The fixed displacement boundary condition has been used by many authors. ${ }^{29,30}$ The positions of the atoms on the border are given according to linear elastic theory and subsequently held fixed as the outer materials are removed during the simulation. Along the $z$ direction, the periodic boundary condition is adopted. But the atoms in the present simulation system can move in the $z$ direction because of six layers of $(11 \overline{2})$ atomic planes adopted. The effects of border conditions on the simulation result were discussed by deCelis et $a l .{ }^{31}$ and others. ${ }^{32}$ The force boundary condition has a significant effect on the process of numerous dislocation emissions. ${ }^{32}$ The deficiency of displacement boundary is that there is no way for a dislocation emitted from the crack tip to penetrate through the border. When the leading dislocation arrives at the boundary, its effect on the boundary will be significant. But the present simulation only concerns the beginning stage of dislocation emission or crack cleavage propagation. Meanwhile our simulation sample has a relatively large size in the $x$ direction. The ligament ahead of the crack tip is about $380 \times(\sqrt{3} / 6) a_{0}$. Hence the emitted dislocations are still far away from the border in our simulation. The effect of border condition on the present simulation results is assumed to be small.

\section{Calculation method}

The leapfrog algorithm ${ }^{33}$ is used in this paper. For the case of the pure shear load, the loading rate is $\dot{K}_{\mathrm{II}}=$ $0.0706 \mathrm{MPa} \mathrm{m}{ }^{1 / 2} / \mathrm{ps}$, and the stress intensity factor $K_{\mathrm{II}}$ is chosen as the loading parameter. For mode I load, the loading rate $\dot{K}_{\mathrm{I}}$ is the same as $\dot{K}_{\mathrm{II}}$. The time step is $1.256 \times 10^{-14} \mathrm{~s}$.

\section{F. Temperature condition}

All atoms in the simulation area are assigned the initial velocities derived from a Maxwellian velocity distribution corresponding to a given temperature. The isothermal process is required, and the total kinetic energy of the whole atomic system remains constant during the simulation. Suppose that the simulation system is connected to a big heat reservoir with a certain temperature which provides enough heat energy to maintain the system at a fixed value throughout the duration of the runs. This means that any small disturbance due to the loading increment or the dislocation emission can be neglected. ${ }^{34,35}$ Here $T=50 \mathrm{~K}$. The result of extending simulation to higher temperature for the metal Mo is given in Ref. 36.

\section{G. Method of determining dislocation position}

Figure 1(e) shows the schematic diagram of the deformed system in which six adjacent $(11 \overline{2})$ atomic planes are projected on the same plane. Edge dislocation can be emitted from the crack tip, and its position is between the atomic planes $L_{A}$ and $L_{B}$ in which atoms are marked by $A_{1}$ and $A_{2}, B_{1}$ and $B_{2}$, etc. Due to the geometric characteristic of the edge dislocation, there is an extra half atomic plane $S_{A}$ of the upper atomic block relative to the lower atomic block along the slip plane. Two conditions should be satisfied in determining the dislocation position in a bcc crystal. First, it is easy for us to calculate $S_{1}$ and $S_{2}$, which are the $x$ components of lines $A_{1} B_{1}$ and $A_{1} B_{2}$, and $\Delta A_{1}$ is defined as follows:

$$
\Delta A_{1}=\left|S_{1}\right|-\left|S_{2}\right|
$$

Similarly $\Delta A_{2}$ can be calculated and so on. If the product of $\Delta A_{1} \times \Delta A_{2}$ is positive, there is no dislocation; otherwise a dislocation might be found. The other condition is that the $x$ component of atom $A_{2}$ corresponds nearly to the midpoint of the $x$ component of line $B_{2} B_{3}$. 


\section{RESULTS AND DISCUSSION}

\section{A. Pure mode II loading}

The positions of the two full dislocations versus the stress intensity factor are plotted in Fig. 2. Little dots in Figs. 2 and 3 identify the positions of partial dislocation associated with the emitted dislocation. As $K_{\mathrm{II}}$ reaches about $0.668 \mathrm{MPa} \mathrm{m}{ }^{1 / 2}$, the first full dislocation has been emitted, and at about $1.17 \mathrm{MPa} \mathrm{m}{ }^{1 / 2}$, the second full dislocation emitted. Because the fixed displacement boundary scheme is used, after a dislocation nucleation, an image force will emerge which always tends to stop the nucleated dislocation moving. Compared to the external load, the image force is smaller, so the nucleated dislocations move away quickly from the crack tip under the repulsive force from the applied $K_{\text {II }}$ stress field. A similar result has been obtained by several authors. ${ }^{23,31,32,37}$ When the dislocation is near the boundary, the effect of the applied $K_{\mathrm{II}}$ stress field on dislocation becomes weak, but the image force becomes larger which finally stops the dislocation moving and causes the pileup of dislocation near the boundary. This is a serious drawback of the fixed displacement scheme which can be eliminated by imposing the force boundary scheme ${ }^{31}$ or the flexible displacement boundary scheme. ${ }^{38,39}$

The curves of dislocation positions versus time are plotted in Fig. 3. Within the distance of $5 a_{0}\left(a_{0}\right.$ is the crystal constant) from the crack tip, the dislocation is in an accelerative state and reaches the constant velocity quickly, about 1800-1950 m/s; see Fig. 3. The interval distance between the first and second full dislocations is about $45 a_{0}$. The first and the second full dislocation have been emitted at $t=10.5$ and $17.5 \mathrm{ps}$, respectively. The time interval is about $7 \mathrm{ps}$. A full dislocation is dissociated into three partial dislocations in the MD simulation.

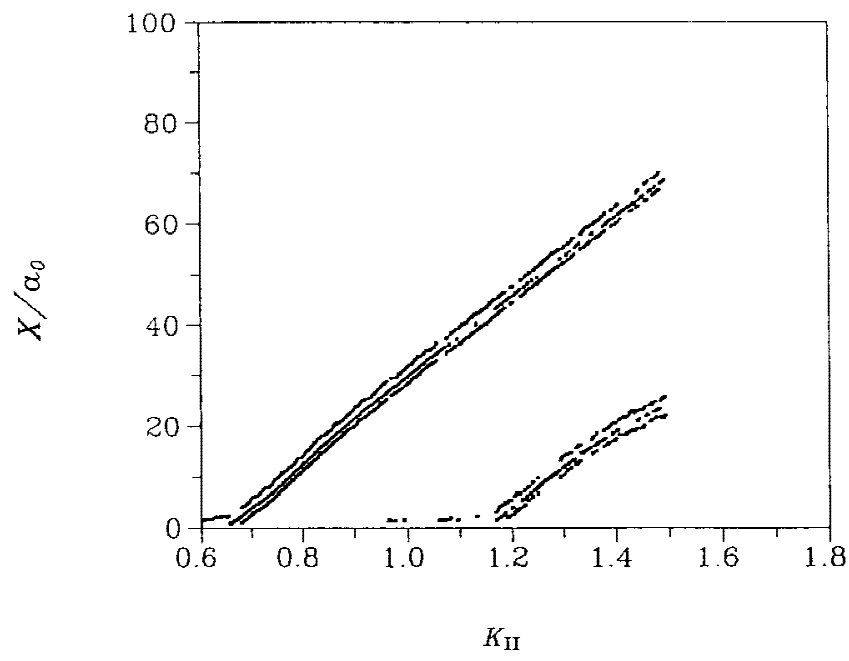

FIG. 2. Dislocation positions versus stress intensity factor under the pure mode II load, with $K_{\mathrm{II}}=1.17 \mathrm{MPa} \mathrm{m}^{1 / 2}$.

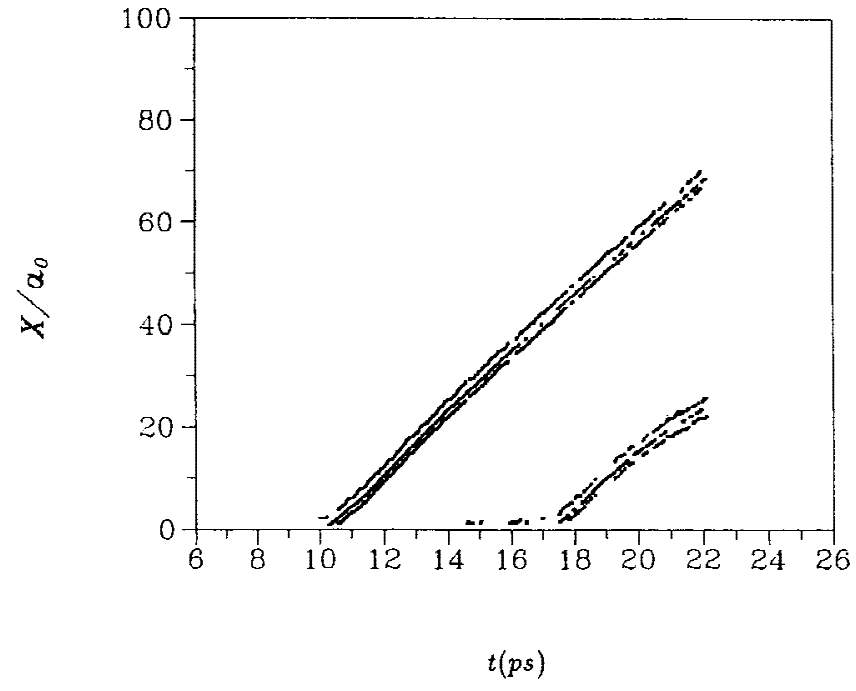

FIG. 3. Dislocation positions versus time under the pure mode II loading.

The moving-away direction of the partial dislocation is not along the [111] $x$ direction but along a line with a certain angle from the $x$ direction on the (110) atomic plane by which the resistance force encountered by partial dislocation is very small. It is easy to determine the moving direction and Burger's vector of full dislocation; they are in the $x$ direction and have a magnitude of $(\sqrt{3} / 2) a_{0}$, respectively, but it is difficult to determine those of partial dislocation, because atoms are in a dynamic state. As $K_{\mathrm{II}}=1.17 \mathrm{MPa} \mathrm{m}^{1 / 2}$, the atom configuration near the crack tip under pure mode II loading is shown in Fig. 4(a), and the two full dislocations (6 partial dislocations) can be observed in Figs. 4(b) and 4(c). The configuration of Fig. 4(a) near the crack tip is similar to Fig. 9 of Ref. 20.

The shear stress distribution along the prolongation of crack plane is shown in Fig. 5 at a load level of $K_{\mathrm{II}}=$ $1.17 \mathrm{MPa} \mathrm{m}{ }^{1 / 2}$. It can be seen that the present result is in good agreement with the elastic solution. The elastic solution is given by Wang, ${ }^{12}$ which takes into account the effect of the emitted dislocation. It is reasonable that the stress near the crack tip given by the elastic solution is greater than that of molecular dynamics (MD).

\section{B. Pure mode I loading}

Except for mode I loading, other conditions such as the loading rate, temperature, size, and crystal orientation of the simulation system are identical to those of the pure mode II loading.

For the convenience of the following analysis, the surface energy $\gamma_{s}$ and the Griffith $K_{\mathrm{Ic}}^{E}$ should be calculated from the " $N$-body" potential which is being used. Finnis and Sinclair $^{40}$ considered the unrelaxed formation energy of the (100) surface and gave the formula for calculating 


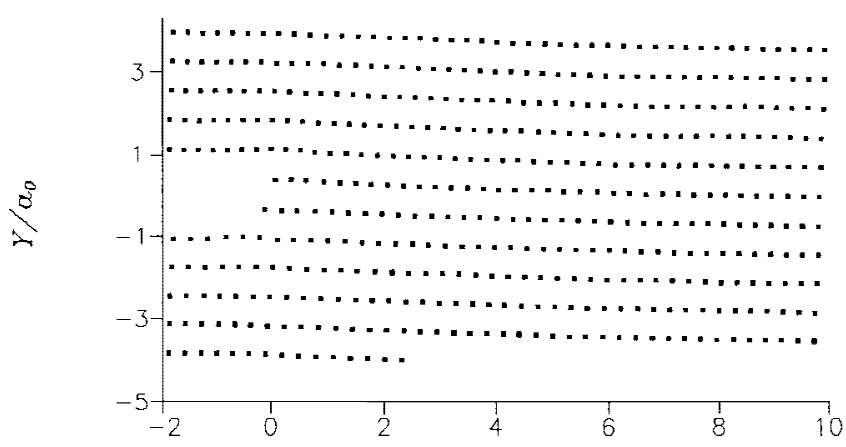

(a)

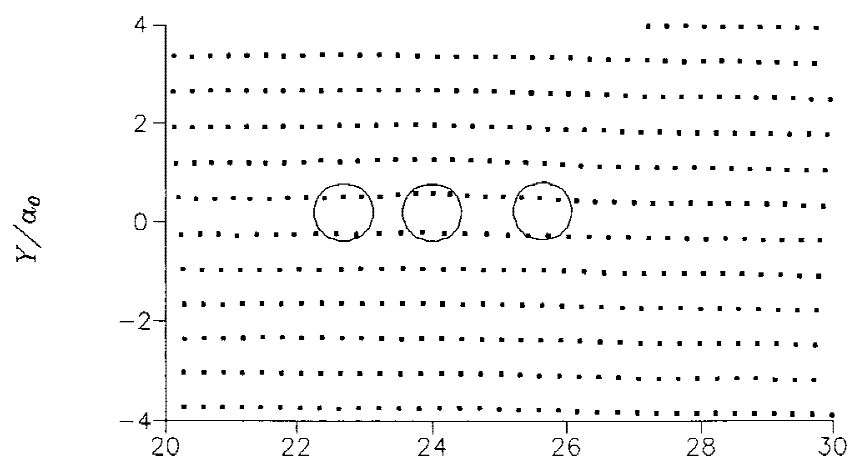

(b)

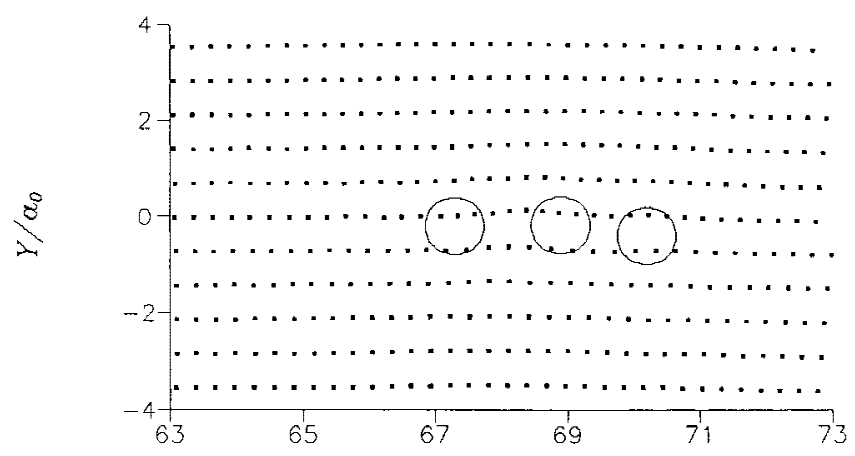

(c)

$$
X / \alpha_{0}
$$

FIG. 4. Atomic configuration near the crack tip under the pure mode II load, with $K_{\mathrm{II}}=1.17 \mathrm{MPa} \mathrm{m}^{1 / 2}$ : (a) atomic configuration near the crack tip; $(b, c)$ atomic configurations of six partial dislocations.

surface energy for the bcc crystal Mo in which the first and second neighbor atoms are counted. Similarly, the energy $\gamma_{s}$ of the $(1 \overline{1} 0)$ surface can be obtained

$$
\begin{aligned}
& \gamma_{s}= \\
& 2\left[\phi\left(r_{1 e}\right)+\phi\left(r_{2 e}\right)+F\left(\frac{8 \phi\left(r_{1 e}\right)+6 \phi\left(r_{2 e}\right)}{\rho_{e}}\right)\right. \\
& \frac{\left.-F\left(\frac{6 \phi\left(r_{1 e}\right)+4 \phi\left(r_{2 e}\right)}{\rho_{e}}\right)-F\left(\frac{6 \phi\left(r_{1 e}\right)+4 \phi\left(r_{2 e}\right)}{\rho_{e}}\right)\right]}{\sqrt{2} a_{0}^{2}}
\end{aligned}
$$

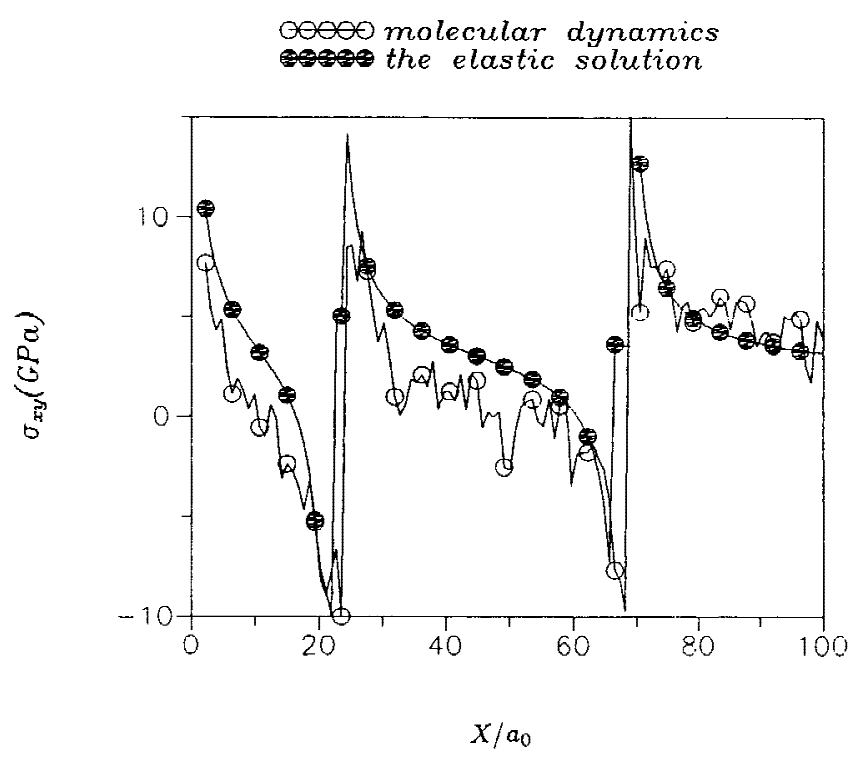

FIG. 5. Shear stress distribution along the prolongation of the crack plane under mode II loading, with $K_{\mathrm{II}}=1.17 \mathrm{MPa} \mathrm{m}{ }^{1 / 2}$.

where all symbols are the same as before. Substituting Eqs. (4) and (5) into Eq. (7), we obtain $\gamma_{s}=2.689 \mathrm{~J} / \mathrm{m}^{2}$. According to fracture theory, the Griffith $K_{\mathrm{Ic}}^{E}$ is

$$
K_{\mathrm{Ic}}^{E}=2 \sqrt{\frac{\mu \gamma_{s}}{1-v}},
$$

where $\mu$ is the Voigt average shear modulus with $\mu=$ $124.8 \mathrm{GPa}$ and $v$ is the Voigt average Poisson ratio with $v=0.2999 .{ }^{41}$ From Eq. (8), we get $K_{\mathrm{Ic}}^{E}=1.385$ $\mathrm{MPa} \mathrm{m}{ }^{1 / 2}$.

For the case of $\theta=0^{\circ}$, the atomic configuration near the crack tip is shown in Figs. 6(a) and 6(b). The initial state of crack propagation is observed clearly when $K_{\mathrm{I}}=$ $1.419 \mathrm{MPa} \mathrm{m}{ }^{1 / 2}$, which is in good agreement with the Griffith $K_{\mathrm{Ic}}^{E}$. When $K_{\mathrm{I}}=1.685 \mathrm{MPa}{ }^{1 / 2}$, the crack propagates about $4 a_{0}$ along the [111] slip direction on the (110) slip plane without any dislocation emission.

The conclusions obtained from the present MD simulation are that the crack behaviors of ductile and brittle in bcc metal Mo are strongly dependent on the loading modes and that the (110) crystal plane of the metal Mo is not only a slip plane under mode II loading but also a cleavage plane under mode I loading. A similar result was also obtained in fcc metal $\mathrm{Cu}^{23}$

As the crack tip moves, we do not move the crack tip field in the boundaries. The present simulation is mainly concerned with the critical $K_{\mathrm{Ic}}$ for the initiation of crack extension and less with the process of crack extension. The crack brittle cleavage is found in Figs. 7-9 for the cases of $\theta=15^{\circ}, 30^{\circ}$, and $45^{\circ}$, respectively. The critical stress intensity factor $K_{\text {Ic }}$ for the initiation of the crack 


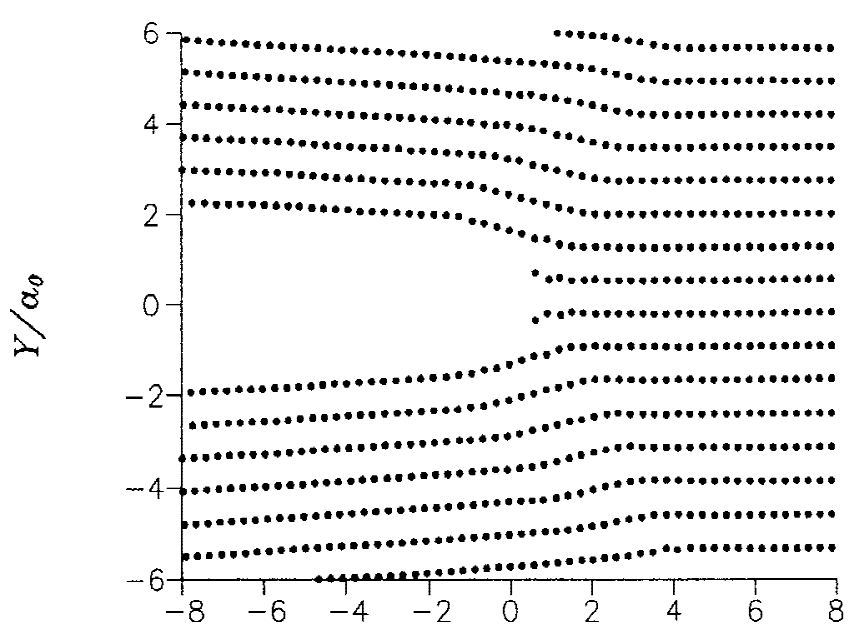

(a)

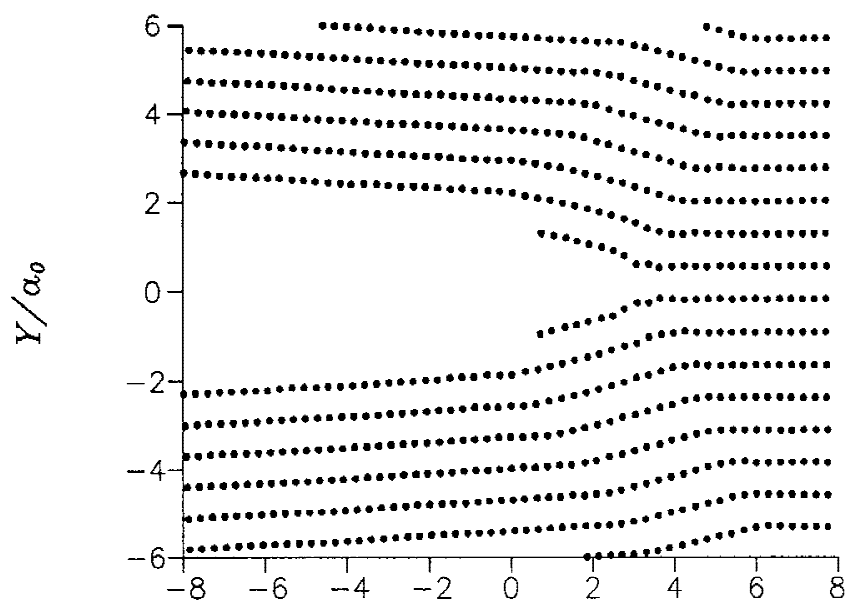

(b)

FIG. 6. Atomic configuration near the crack tip under mode I loading, with $\theta=0^{0}$ : (a) $K_{\mathrm{I}}=1.419 \mathrm{MPa} \mathrm{m}^{1 / 2}$; (b) $K_{\mathrm{I}}=1.685 \mathrm{MPa} \mathrm{m}^{1 / 2}$.

propagation increases with increasing $\theta$; they are 1.464 , 1.552 , and $1.685 \mathrm{MPa} \mathrm{m}^{1 / 2}$, respectively. A common characteristic of four simulations of $0^{\circ}, 15^{\circ}, 30^{\circ}$, and $45^{\circ}$ is that the cleavage propagation is along the (110) slip plane without any dislocation emission. Three factors may be mentioned concerning this result. First, temperature given in our simulation is quite lower, $T=50 \mathrm{~K}$, which is not enough to motivate dislocation emission. Second, both the pure mode I loading and periodicity boundary condition along the $z$ direction are adopted which reduce the possibility of screw dislocation emission. Third, the situation is different in fcc metal $\mathrm{Cu}$; as $\theta=16^{\circ}$ and $25^{\circ}$, the crack propagates, accompanying a number of dislocation emissions. This implies that the dislocation mobility in bcc metal Mo is less than that of fcc metal $\mathrm{Cu}^{23}$

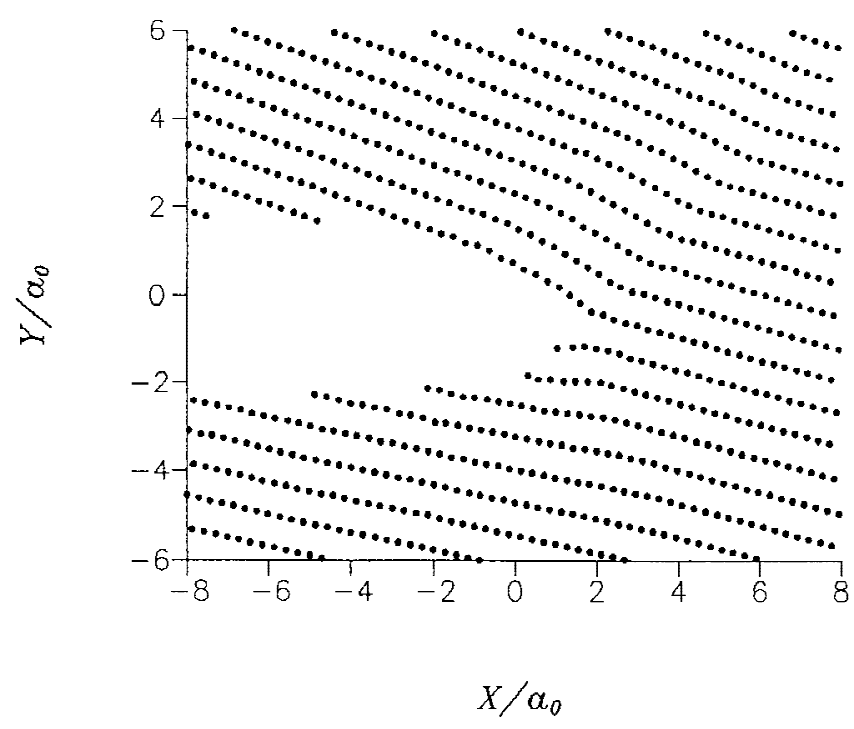

FIG. 7. Atomic configuration near the crack tip, under mode I loading, with $\theta=15^{\circ}$ and $K_{\mathrm{I}}=1.464 \mathrm{MPa} \mathrm{m}^{1 / 2}$.

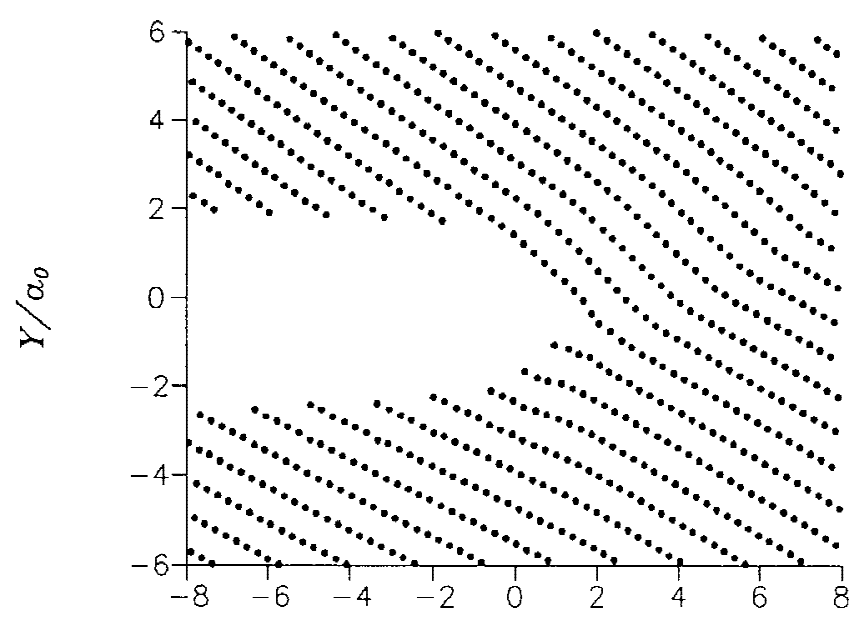

$$
X / \alpha_{0}
$$

FIG. 8. Atomic configuration near the crack tip under the pure mode I loading, with $\theta=30^{\circ}$ and $K_{\mathrm{I}}=1.552 \mathrm{MPa} \mathrm{m}^{1 / 2}$.

On the basis of linear elastic fracture theory, the following expressions can be obtained under mode I or II loading, respectively: ${ }^{12}$

$$
\begin{aligned}
& K_{\mathrm{I}}^{*}=1 / 2 K_{\mathrm{I}} \cos (\theta / 2)[1+\cos (\theta)]=f_{\mathrm{I}}(\theta) K_{\mathrm{I}} \\
& K_{\mathrm{II}}^{*}=1 / 2 K_{\mathrm{I}} \cos (\theta / 2) \sin (\theta)=f_{\mathrm{II}}(\theta) K_{\mathrm{I}} \\
& K_{\mathrm{I}}^{*}=-3 / 2 K_{\mathrm{II}} \cos (\theta / 2) \sin (\theta) \\
& K_{\mathrm{II}}^{*}=1 / 2 K_{\mathrm{II}} \cos (\theta / 2)\left[3 \cos \left(\theta^{\prime \prime \prime \prime}-1\right] .\right.
\end{aligned}
$$

Here $K_{\mathrm{I}}$ and $K_{\mathrm{II}}$ are the stress intensity factors in $(\mathrm{OXY})$, and $K_{\mathrm{I}}^{*}$ and $K_{\mathrm{II}}^{*}$ are in the coordinate system $\left(O X^{*} Y^{*}\right)$. 


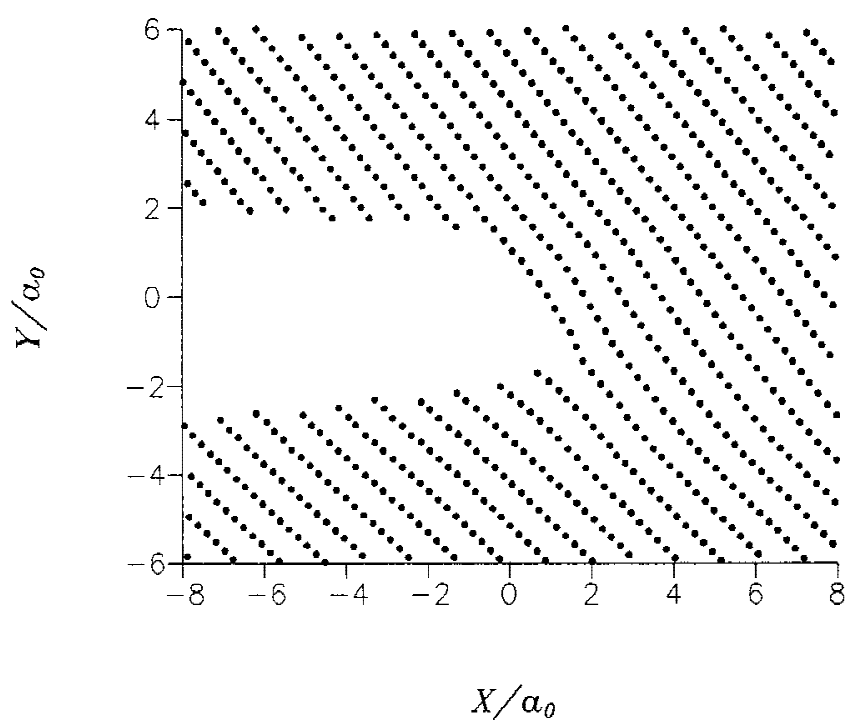

FIG. 9. Atomic configuration near the crack tip under mode I loading, with $\theta=45^{\circ}$ and $K_{\mathrm{I}}=1.685 \mathrm{MPa} \mathrm{m}^{1 / 2}$.

The $X^{*}$ axis is coincident with the [111] slip direction and $Y^{*}$ axis is perpendicular to the (110) slip plane; see Figs. 1(a) and 1(d). The stress intensity factors $K_{1}^{*}$ corresponds to the crack cleavage behavior, and $K_{\mathrm{II}}^{*}$ causes the plastic shear along the slip plane. When the crack branches into the [111] slip direction, the branched crack is subjected to mixed-mode loading. The energy release rate $G$ can be approximately evaluated by the following formula: ${ }^{12,40}$

$$
G=\frac{1-v^{2}}{E}\left(K_{I}^{*^{2}}+K_{I I}^{*^{2}}\right)
$$

Hence we introduce an effective stress intensity factor

$$
\left(K_{\mathrm{I}}^{*}\right)_{e f}=\sqrt{\left(K_{\mathrm{I}}^{*}\right)^{2}+\left(K_{\mathrm{II}}^{*}\right)^{2}} \geqslant K_{\mathrm{Ic}}^{M}
$$

As $\theta=0^{\circ}$, the first full dislocation is emitted from the crack tip at $K_{\text {IIe }}=0.668 \mathrm{MPa} \mathrm{m}^{1 / 2}$ under pure mode II loading and the crack propagates at the stress level of $K_{\mathrm{Ic}}^{M}=1.419 \mathrm{MPa} \mathrm{m}^{1 / 2}$ under pure mode I loading. Both $K_{\mathrm{IIe}}$ and the $K_{\mathrm{Ic}}^{M}$ are chosen as the reference values in the following analysis.

For the case of $\theta=45^{\circ}$, as the external stress intensity factor $K_{\mathrm{I}}$ reaches $1.685 \mathrm{MPa} \mathrm{m}{ }^{1 / 2}$, from Eq. (9a) we obtain $K_{\mathrm{I}}^{*}=1.329 \mathrm{MPa} \mathrm{m}^{1 / 2}$ and $K_{\mathrm{II}}^{*}=0.549 \mathrm{MPa} \mathrm{m}^{1 / 2}$. Because $K_{\mathrm{II}}^{*}$ is less than $K_{\mathrm{II}}$, the result of no dislocation emission from crack tip can be understood and $K_{I}^{*}$ is less than $K_{\mathrm{Ic}}^{M}$, but $\left(K_{\mathrm{I}}^{*}\right)_{e f}=1.439 \mathrm{MPa} \mathrm{m}{ }^{1 / 2}$, which is greater than $K_{\mathrm{Ic}}^{M}$.

Due to the contribution of $\left(K_{\mathrm{I}}^{*}\right)_{e f}$, the crack cleavage propagation can be observed in Fig. 9. A similar analysis can be applied to cases of $\theta=15^{\circ}$ and $30^{\circ}$; see Table II. This implies that the present simulation result meets the cleavage criterion. ${ }^{11}$
TABLE II. Values of the functions $f_{\mathrm{I}}(\theta)$ and $f_{\mathrm{II}}(\theta)$ and stress intensity factors are listed. $K_{\mathrm{I}}$ is the external load, and $K_{\mathrm{I}}^{*}, K_{\mathrm{II}}^{*}$, and $\left(K_{\mathrm{I}}^{*}\right)_{e f}$ are obtained from Eqs. (9a) and (11). The unit of the stress intensity factors is $\mathrm{MPa} \mathrm{m}^{1 / 2}$.

\begin{tabular}{rcccccc}
\hline \hline$\theta^{0}$ & $f_{\mathrm{I}}(\theta)$ & $f_{\mathrm{II}}(\theta)$ & $K_{\mathrm{I}}$ & $K_{\mathrm{I}}^{*}$ & $K_{\mathrm{II}}^{*}$ & $\left(K_{\mathrm{I}}^{*}\right)_{e f}$ \\
\hline 0 & 1.000 & 0.000 & 1.419 & 1.419 & 0.000 & 1.419 \\
15 & 0.975 & 0.128 & 1.464 & 1.427 & 0.187 & 1.439 \\
30 & 0.901 & 0.241 & 1.552 & 1.398 & 0.374 & 1.447 \\
45 & 0.789 & 0.326 & 1.685 & 1.329 & 0.549 & 1.438 \\
\hline \hline
\end{tabular}

According to our simulated results of the pure metal Mo, under mode I loading, the brittle cleavage of the slight blunted crack is observed; see Figs. 6-9. When the blunting crack becomes sharp, the stress singularity is increased near the crack tip and crack cleavage propagation would be easier than that of the present simulation; it can be deduced that the change of deformation mode from brittle cleavage to ductile is not possible. And under mode II loading, when the slightly blunted crack becomes sharp and the crack plane is coincident with the slip plane, because there is not any tension stress component along the crack plane, the crack extension would not take place, so we can confirm that the change of deformation mode from ductile to brittle is also not possible.

\section{CONCLUSION}

We have examined the crack tip behaviors of brittle and ductile in pure Mo. The crack propagation is a response to mode I loading, and dislocation emission, to mode II loading. Criteria for predicting brittle or ductile behavior can be characterized in terms of the stress intensity factors. The main results are as follows:

(1) The simulation in MD shows that the (1 $\overline{1} 0)$ atomic plane is not only a slip plane but also a crack cleavage plane at a temperature of $50 \mathrm{~K}$. The dislocation emission is observed along the slip plans under pure mode II loading, but the crack propagates along the same slip plane under pure mode I loading.

(2) The result obtained under mode II loading shows that the two full dislocations have been emitted from the crack tip at stress level of $K_{\text {IIe }}=1.17 \mathrm{MPa} \mathrm{m}^{1 / 2}$ without any crack extension. The shear stress distribution ahead of the crack tip given by the present MD simulation under mode II loading is in good agreement with the elastic solution which takes into account the effect of the dislocation. The shear stress of elastic solution near the crack tip is slightly greater than that of MD.

(3) Under mode I loading, the critical stress intensity factor for crack initiation given by the present MD simulation is in good agreement with the Griffith $K_{\mathrm{Ic}}^{E}$, which is obtained from the calculation of surface energy $\gamma_{s}$ based on the " $N$-body" potential. 
(4) The effect of crystal orientation on crack initiation and dislocation emission can be demonstrated on the basis of stress intensity factor theory. The crack initiation is controlled by the effective stress intensity factor $\left(K_{I}^{*}\right)_{e f}$, and the dislocation emission is controlled by $K_{\mathrm{II}}^{*}$.

\section{ACKNOWLEDGMENTS}

The valuable comments given by the reviewers are gratefully acknowledged. The research presented here was supported by the National Natural Science Foundation of China (No. 19704100) and the National Science Foundation of the Chinese Academy of Sciences (Project KJ951-1-201).

\section{REFERENCES}

1. A.A. Griffith, Philos. Trans. R. Soc. London A 221, 163 (1920).

2. Y.H. Chiao and D.R. Clarke, Acta Metall. 47, 203 (1989).

3. A. George and G. Michot, Mater. Sci. Eng. A 164, 118 (1993).

4. S.M. Ohr, Mater. Sci. Eng. 72, 1 (1985).

5. J.S. Wang and P.M. Anderson, Acta Metall. 39, 779 (1991).

6. K. Hiroaki, Q. Akira, K. Haruyoshi, and Y. Hideo, Trans. Jpn. Inst. Met. 26, 341 (1985).

7. G.E. Beltz and J.S. Wang, Acta Metall. 40, 1675 (1992).

8. H. Zhang, A.H. King, and R. Thomson, J. Mater. Res. 6, 314 (1991).

9. G. Schoeck, Philos., Mag. A. 74, 419 (1991).

10. J.R. Rice, J. Mech. Phys. Solids 40, 239 (1992).

11. J.R. Rice, G.E. Beltz, and Y. Sun, in Topics in Fracture and Fatigue, edited by A.S. Argon (Springer, Berlin, 1992), pp. 1-58.

12. T.C. Wang, Philos. Mag. A 74, 4, 983 (1996).

13. J.R. Rice, R. Thomson, Philos. Mag. 29, 73 (1974).

14. G. Xu and M. Ortiz, Int. J. Numer. Methods Eng. 36, 3675 (1993).

15. G. Xu and A.S. Argon, Philos. Mag. A 72, 415 (1995).

16. G. Xu, A.S. Argon, and M. Ortiz, Philos. Mag. A 75, 341 (1997).

17. S.J. Zhou, A.E. Carlsson, and R. Thomson, Phys. Rev. Lett. 72, 852 (1994)

18. L.G. Orlov, Sov. Phys. Solid State 9, 1836 (1968).

19. F. Cleri, S. Yip, D. Wolf, and S. Phillpot, Phys. Rev. Lett. 79, 1309 (1997).
20. J. Schiøtz, L.M. Canel, and A.E. Carlsson, Phys. Rev. B 55, 6211 (1997).

21. M. Mullins, Int. J. Fract. 24, 189 (1984).

22. R.G. Hoagland, M.S. Daw, S.M. Foiles, and M.I. Baskes, J. Mater. Res. 5, 313 (1990).

23. Y.W. Zhang, T.C. Wang, and Q.H. Tang, Scr. Metall. Mater. 33, 267 (1995).

24. Q.H. Tang, T.C. Wang, and Y.W. Zhang, Acta Mech. Sin. 12, 358 (1996).

25. M.S. Daw and M.I. Baskes, Phys. Rev. B 29, 6443 (1984).

26. R.A. Johnson and D.J. Oh, J. Mater. Res. 4, 1195 (1989).

27. Landolt-Bornstein, Numerical Data and Functional Relationships in Science and Technology, New Series, Group III (SpringerVerlag, Berlin, 1979), Vol. 2.

28. P. Gumbsch, J. Mater. Res. 10, 2897 (1995).

29. P.C. Gehlen and M.F. Kanninen, in Inelastic Behavior of Solids, edited by M.F. Kanninen, W.F. Alder, A.R. Rosenfield, and R.I. Jaffee (McGraw-Hill, New York, 1970), p. 587.

30. M.F. Kanninen and P.C. Gehlen, Int. J. Fract. Mech. 1, 471 (1971).

31. B. deCelis, A.S. Argon, and S. Yip, J. Appl. Phys. 54, 4664 (1983).

32. H. Kitagawa, A. Nakatani, and Y. Shibutani, in Proceedings of the IMMM '93, International Seminar Microstructures and Mechanical Properties of New Engineering Materials, edited by M. Tokada, B. Xu, and M. Senoo (Academic Press, Mie University, Mie, Japan, 1993).

33. D.W. Heermann, Computer Simulation Methods in Theoretic Physics, 2nd ed. (Springer-Verlag, Berlin, 1990).

34. S. Nose, in Computer Simulation in Materials Science, edited by M. Meyer and V. Pontikis (1991), p. 21.

35. I. Alber, J.L. Bassani, M. Khantha, V. Vitek, and G.J. Wang, Phil. Trans. R. Soc. Lond. A 339, 555 (1992).

36. Q.H. Tang and T.C. Wang, Acta Mech. Solids Sin. 11, 189 (1998).

37. H. Tan, W. Yang, Acta Mech. Sin. 10, 150 (1994).

38. R.G. Hoagland, J.P. Hirth, and P.C. Gehlen, Philos. Mag. 34, 413 (1976).

39. J.E. Sinclair, P.C. Gehlen, R.G. Hoagland, and J.P. Hirth, J. Appl. Phys. 49, 3890 (1978).

40. M.W. Finnis and J.E. Sinclair, Philos. Mag. A 50, 45 (1984).

41. D.I. Bolf and J. de Klerk, J. Appl. Phys. 33, 2311 (1962). 